\title{
Predictive role of haemoglobin on disease response to neoadjuvant chemotherapy in breast cancer
}

Claudia Omarini ${ }^{1}$, Elena Iattoni ${ }^{1}$, Maria Elisabetta Filieri ${ }^{1}$, Angela Toss ${ }^{1}$, Giulia Grizzi ${ }^{1}$, Antonella Tamma $^{1}$, Cinzia Del Giovane ${ }^{1}$, Stefano Cascinu ${ }^{1}$ and Federico Piacentini ${ }^{1}$

University Hospital of Modena and Reggio Emilia ${ }^{1}$

Background: Tumour hypoxia has been shown to play an important role in the outcome of cancer patients. Data on the predictive role of haemoglobin $(\mathrm{Hb})$ on disease response to primary therapies in breast cancer (BC) are lacking. The purpose of this study is to evaluate the influence of Hb level throughout treatment course in predicting the response to neoadjuvant chemotherapy.

Methods: 252 patients diagnosed with stage I-III BC treated with anthracycline-taxane based primary chemotherapy were evaluated. Patient and tumor characteristics and treatment information were collected. Standard biological parameters (Ki67, nuclear grade, hormone receptors and HER2 status) were correlated with pathologic complete response (pCR). We focused on $\mathrm{Hb}$ (baseline and after therapy levels, drop in $\mathrm{Hb}$ throughout treatment) and its correlation to $\mathrm{pCR}$ rate. The Hb cut-off to discriminate anaemic vs non-anaemic patients was set at $12,0 \mathrm{~g} / \mathrm{dl}$.

Results: Globally, pCR was achieved in 58 patients (23\%), mainly in case of younger age ( $<50$ years $=29 \%, \geq 50$ years $=17 \% ; \mathrm{p}=0,01)$, high nuclear grade $($ Grade $1-2=0 \%$, grade $3=27 \% ; \mathrm{p}<0,0001)$, high ki67 $(\geq 20 \%=26 \%,<20 \%=12 \% ; p=0,02)$ and hormone receptor negative status (Luminal $\mathrm{B} / \mathrm{HER} 2$ negative $=9 \%$, Luminal B/HER2 positive $=32 \%$, HER2 enriched $=46 \%$, triple negative $=$ $37 \%$; $<0,0001$ ). Median baseline $\mathrm{Hb}$ was $13,3 \mathrm{~g} / \mathrm{dl}$ while median $\mathrm{Hb}$ level after chemotherapy was 11,6 g/dl: pCR was not influenced by $\mathrm{Hb}$ level before and after primary chemotherapy. No difference in $\mathrm{Hb}$ levels were observed stratifying patients according to nuclear grade, tumour stage and cancer subtypes. Anaemia due to chemotherapy was reported in $56 \%$ of patients. The decrease in $\mathrm{Hb}$ levels from baseline was greater in patients with lower response rate. On univariate analysis, a decrease in $\mathrm{Hb} \geq 2 \mathrm{~g} / \mathrm{dl}$ was associated with a significantly lower rate of pCR (15\% vs $43 \% ; \mathrm{p}=0,047)$. This correlation was even more evident in the subgroup of anaemic patients $(17 \%$ vs $32 \%$; $=0,037)$.

Conclusions: A decrease in $\mathrm{Hb} \geq 2 \mathrm{~g} / \mathrm{d}$ during neoadjuvant chemotherapy may negatively affect the rate of $\mathrm{pCR}$ in $\mathrm{BC}$ patients, thus suggesting that anaemia should be avoided in order to obtain the best response to primary treatments. 